\title{
«Mujer de mi madre». Identidad y funciones de los coprogenitores y de las coprogenitoras en familias reconstituidas no heteronormativas
}

\author{
Gloria Álvarez Bernardo \\ Nuria Romo Avilés \\ Universidad de Granada. Instituto Universitario de Estudios de las Mujeres y de Género. \\ Departamento de Antropología Social y Cultural \\ gloab@ugr.es; nromo@ugr.es
}

\section{Resumen}

En este artículo se aborda la figura de los coprogenitores y de las coprogenitoras en las familias reconstituidas no heteronormativas. Se analiza tanto su identidad como los roles que desempeña dentro del conjunto de las relaciones familiares. Los datos proceden de cinco entrevistas semiestructuradas realizadas a familias reconstituidas no heteronormativas que provenían tanto de una relación heterosexual previa (4) como de una relación no heterosexual previa (1). Las personas entrevistadas coinciden en señalar que la opción sexual no es fuente de conflicto entre sus hijos e hijas, si bien el divorcio y el inicio de una nueva relación es lo que puede provocar problemas económicos, parentales o personales, entre otros. Por ello, es importante definir la posición del coprogenitor o de la coprogenitora ante esta nueva situación. En lo relativo a la identidad, el coprogenitor o la coprogenitora se define y es definido como un amigo de los hijos y/o de las hijas de su pareja. Y en cuanto a sus funciones, se limitan a darle consejos sobre el cuidado y la educación de sus criaturas. Traspasar estos límites puede ser fuente de conflicto familiar. Así mismo, la presencia y la participación del otro progenitor, la edad de los hijos y/o de las hijas o que exista convivencia son factores que influyen en la definición de la identidad y de las funciones de los coprogenitores y de las coprogenitoras.

Palabras clave: parentesco; estructura familiar; trayectoria familiar; parejas del mismo sexo; divorcio 
Abstract. "My mother's wife": Identity and functions of co-parents in non-heteronormative stepfamilies

This article examines the representation of stepparents in non-heteronormative stepfamilies and addresses their identity and roles. Four families that came from a prior heterosexual marriage and one from a prior homosexual marriage were interviewed. The results reveal that sexual orientation is not relevant in family relationships for the couples interviewed. However, divorce and a new relation can be a cause of conflict, making it important to define the role of the stepparents. Stepparents are defined as friends and counsellors, roles they should not overstep. Moreover, involvement of the other parent, the children's age and cohabitation are factors that influence stepparents' identity and functions.

Keywords: kinship; family structure; family trajectories; same-sex families; divorce

\begin{tabular}{|c|c|}
\hline \multicolumn{2}{|c|}{ Sumario } \\
\hline 1. Transformaciones en los vínculos & 2. Metodología \\
\hline de parentesco de las familias españolas. & 3. Resultados \\
\hline $\begin{array}{l}\text { Aacia una nueva normatividad en la } \\
\text { construcción de los hogares }\end{array}$ & 4. Discusión y conclusiones \\
\hline & Referencias bibliográfícas \\
\hline
\end{tabular}

\section{Transformaciones en los vínculos de parentesco de las familias españolas. Hacia una nueva normatividad en la construcción de los hogares}

Los últimos años se han caracterizado por una serie de transformaciones sociales y políticas que han favorecido la emergencia de múltiples modelos familiares. Estos modelos cuestionan algunos de los principios del parentesco tal y como ha sido conceptualizado en Occidente (Rivas, 2008). En España, la aprobación de la Ley del divorcio de 1981 ha supuesto que las personas divorciadas puedan iniciar una nueva relación de pareja y aportar, cuando los hay, hijas e hijos a un nuevo proyecto familiar. Por su parte, la lucha del movimiento LGTBI (lesbianas, gays, transexuales, bisexuales e intersexuales) ha favorecido el avance en los derechos familiares (matrimonio, adopción y acceso a las técnicas de reproducción asistida, entre otros), así como la visibilidad de los modelos de familia encabezados por estas personas (Mezey, 2008; Pichardo, 2011). La combinación de estos factores ha tenido como resultado la emergencia de nuevos modelos familiares. En lo que respecta a los hogares no heteronormativos combinan, por un lado, un proceso de reconstitución familiar en el que se aportan hijos y/o hijas de una relación heterosexual previa $y$, por otro, la opción sexual de la pareja que encabeza la nueva unidad familiar no se ajusta a la norma heterosexual ${ }^{1}$.

1. Las cifras sobre familias reconstituidas encabezadas por parejas del mismo sexo son muy limitadas, por lo que se desconoce la magnitud del fenómeno. El Censo de Población de 
En el contexto español, no existen investigaciones específicas sobre estas nuevas tipologías familiares, si bien las referencias a estas familias se integran en estudios más generales sobre parentesco (López, 2014; Pichardo, 2008). Este hecho concuerda con la escasa investigación que hay, en general, sobre familias no heteronormativas (Donovan, 2000; Mallon, 2004; Patterson, 2000) y que se agudiza en el caso español (Pichardo, 2008). Ante este panorama, es preciso descifrar cuáles son las características de los procesos de reconstitución familiar, así como las peculiaridades que comporta la opción sexual de la pareja adulta. A diferencia de otros procesos de formación familiar no heteronormativos, en los que la maternidad y la paternidad son decisiones muy meditadas (Berkowitz y Marsiglio, 2007; Imaz, 2006; Pichardo, 2009), las familias reconstituidas se caracterizan porque la nueva pareja de la progenitora o del progenitor no ha participado en esta decisión (Brown y Perlesz, 2008; Hequembourg, 2004; Herrera, 2009; Mezey, 2008; Tasker, 2013; Wright, 2011). De este modo, el proceso de reconstitución puede convertirse en una fuente de conflicto, tanto para la pareja (Current-Juretschko y Bigner, 2005) como para los hijos e hijas que pasan a formar parte de este proyecto familiar (Patterson, 2000). Como nos recuerda Roni Berger (2000), solo hace falta acudir a la literatura infantil para comprobar que esta se encuentra plagada de ejemplos que presentan a la madrastra, principalmente, como un ser perverso y maligno.

Por tanto, en la reconstitución familiar perviven y aparecen personas que definen y redefinen identidades, roles y funciones dentro de un complejo entramado de relaciones que autoras como Elisabeth Beck-Gernsheim (2000: 35) denominan "constelaciones familiares». Dentro de estas constelaciones, se ha privilegiado la relación, y consecuentemente la investigación, entre los hijos e hijas y sus progenitores y progenitoras, pero poco se ha dicho acerca de la nueva pareja de la madre o del padre que permanece como un «miembro invisible» (Tasker, 2013: 9).

Esa invisibilidad social y teórica se muestra en la ausencia de un término específico con el que designar a estas personas (Brown y Perlesz, 2008; Erera y Fredriksen, 2001). En este sentido, la investigación que Janet Wright (2011) realizó con familias reconstituidas encabezadas por parejas de mujeres menciona tres términos que resultan del grado de implicación y reconocimiento de esta persona en el proyecto familiar. Para esta autora, la comadre es la que más cercana se encuentra a la figura materna, tantos por los roles que desempeña como por el reconocimiento del que goza por parte de todos los miembros de la unidad familiar. En una posición intermedia se encuentra la madrastra. Por

2011 estima que el 99,5\% del medio millón de familias reconstituidas que residen en España están formadas por parejas heterosexuales. Por lo que respecta a los hogares formados por parejas del mismo sexo, la Encuesta Continua de Hogares (ECH) señala que en 2015 había 93.400 hogares, de los cuales 40.500 estaban constituidos por mujeres y 52.900, por hombres. Este número se aleja del ofrecido por el Censo del año 2011, que cifraba dichos hogares en 54.920. Los datos más actuales relativos a parejas del mismo sexo con hijos e hijas son los del Censo de 2011. En aquel momento había 5.890 hogares de este tipo, de los que 2.490 estaban encabezados por hombres y 3.400 , por mujeres. 
último, la coprogenitora sería quien estaría más alejada de los roles parentales y que Wright describe en los siguientes términos: «[...] un ayudante, un soporte y una consejera de la madre biológica, una pariente activa del niño (aunque a veces esto es rebatido) y un miembro de la familia entregado y comprometido» (2011: 128).

Aunque en la literatura científica española sobre familias reconstituidas se suele emplear el término madrastra o padrastro (Jociles y Villaamil, 2008; Rivas, 2008, 2012), en el trabajo que aquí se presenta se ha optado por los términos coprogenitor y coprogenitora. En primer lugar, porque el perfil de estas personas se ajusta a la propuesta de Wright (2011) y, en segundo lugar, conforme al Diccionario de la lengua española de la Real Academia, el prefijo co- significa 'reunión, cooperación, agregación'. Algunas de estas cualidades se corresponden, como se expondrá a continuación, con las funciones desempeñadas por los coprogenitores y las coprogenitoras, en la medida en que comparten espacios y ayudan y suman experiencias a la nueva unidad familiar constituida.

Junto con la indefinición terminológica, otro de los retos a los que deben enfrentarse los coprogenitores y las coprogenitoras es la ausencia de "guiones» a partir de los cuales puedan definir su rol respecto a los hijos y/o hijas de su pareja. A esto se suma una idea de duplicidad genérica. En este sentido, el coprogenitor o la coprogenitora debe afrontar sus funciones de forma conjunta con el progenitor y la progenitora de su mismo sexo lo que, dentro de un esquema de género estricto, puede convertirse en un hándicap a la hora de definir y consensuar cuáles han de ser sus funciones y responsabilidades (Erera y Fredriksen, 2001; Hequembourg, 2004).

Retomando este último punto, la opción sexual de las coprogenitoras y los coprogenitores es otro de los desafíos a los que están sujetas las familias reconstituidas no heteronormativas. Su opción, alejada de la norma heterosexual, puede llegar a ser un motivo de rechazo y de exclusión, tanto dentro como fuera de la unidad familiar (Berger, 2000; Hequembourg, 2004; Lynch, 2005; Tasker, 2013). En consecuencia, estas familias se enfrentan a una doble dificultad si son comparadas con los hogares heterosexuales reconstituidos:

El proceso de «llegar a ser una familia» es más complicado para las familias reconstituidas de lesbianas y gays. No sólo tienen que aprender cómo llegar a ser una familia reconstituida como las familias reconstituidas heterosexuales $\mathrm{y}$ afrontar la ilegitimidad de sus relaciones igual que las parejas de lesbianas y gays, a diferencia de ambos, tienen que decidir en el contexto familiar si salen del armario, con quién y cómo afrontar las consecuencias de tal decisión. (Lynch y Murray, 2000: 4)

Ese proceso al que se refieren Jean Lynch y Kim Murray relativo a las consecuencias de «salir del armario» está directamente relacionado con el peso que la norma heterosexual puede llegar a ejercer sobre estas familias. Esa norma es lo que autores como Michael Warner denominan «heteronormatividad», según la cual 
[...] la pareja heterosexual representa el principio de la unión social en sí. En el pensamiento social este principio es típicamente mediado a través de conceptos tales como dependencia y reproducción, y es entonces naturalizado a través de diferentes y sofisticados trabajos. (Warner, 2001: XXI)

En la medida en que estas familias se alejan de la heteronormatividad se enfrentan a otra serie de prejuicios. Por un lado, algunas de estas personas acaban en una unión heterosexual debido a la presión social a la que se sienten sometidas y al peso que ciertas instituciones, como el matrimonio heterosexual, tienen en nuestro contexto: «el matrimonio heterosexual como una trayectoria vital asumida [...] un requisito para construir una vida exitosa y plena» (Wolkomir, 2009: 503). Así mismo, para otras personas, el matrimonio o la unión heterosexual ha sido y es la única opción para satisfacer sus deseos de paternidad y de maternidad (Pichardo, 2008).

Otro de los prejuicios que algunas de estas familias deben afrontar respecto a la influencia ejercida por la heteronormatividad está relacionado con una concepción de la opción sexual como algo variable, en función de las circunstancias personales o sociales (Blackwood y Wieringa, 2003; Cucchiari, 2000; Lynch, 2005). Esto supone un reto para la construcción de la heterosexualidad como una entidad en donde los deseos se conducen de forma estática y binaria (Ingraham, 1994; Lehr, 1999; Weston, 2003). De este modo, encontrar a una persona de su mismo sexo que supla las carencias de su relación heterosexual puede ser motivo suficiente para finalizar dicha relación.

Por tanto, las familias reconstituidas heteronormativas contravienen dos de los principios característicos del parentesco occidental. En primer lugar, se produce una ruptura en la continuidad de los vínculos conyugal y filial (Beck-Gernsheim, 2002; Rivas, 2008) y entre la sexualidad y la reproducción (Cadoret, 2013; Lehr, 1999; Pichardo, 2008). El objetivo de este artículo es saber cuál es la posición de los coprogenitores y de las coprogenitoras en las familias no heteronormativas. En concreto, intentar conocer cómo se definen y cómo son definidos o definidas por el resto de miembros de la unidad familiar y cuáles son las funciones que desempeñan dentro del entramado del hogar.

\section{Metodología}

El trabajo que aquí se presenta forma parte de un proyecto de investigación más amplio que tiene por objetivo conocer la realidad de las familias no heteronormativas en el contexto español ${ }^{2}$. Dentro de este proyecto, se realizaron un total de veintiuna entrevistas semiestructuradas a familias no heteronormativas con distintos procesos de constitución familiar (reconstituidas, adoptivas y resultantes de tratamientos de reproducción asistida). En el presente artículo se exponen los resultados de la explotación del contenido de cuatro entrevistas

2. Esta investigación se ha desarrollado dentro de la convocatoria de becas de Formación de Profesorado Universitario del Ministerio de Educación (España). Identificación AP-2010-1707. 
Tabla 1. Características sociodemográficas de la muestra

\begin{tabular}{lllcc}
\hline Informantes & Edad & Estudios & Ingresos & Hijos y/o hijas \\
\hline Diego & D: 44 & D: diplomatura & $3001-4500$ & 2 \\
Rodrigo & R: 52 & R: diplomatura & & \\
\hline Olga & O: 49 & O: graduado escolar & $1801-2400$ & 2 \\
Tamara & T: 45 & T: formación profesional & & \\
\hline Lorena & L: 59 & L: diplomatura & $1801-2400$ & 1 \\
Diana & D: 54 & D: licenciatura & & \\
\hline Enrique & E: 28 & E: formación profesional & $3001-4500$ & 1 \\
Simón & S: - & S: licenciatura & & 2 \\
Virginia & V: 57 & V: formación profesional & $3001-4500$ & 2 \\
Dolores & D: 50 & D: licenciatura & & \\
\hline
\end{tabular}

Fuente: elaboración propia.

semiestructuradas realizadas a parejas del mismo sexo que encabezan familias reconstituidas procedentes de una relación heterosexual previa, así como a una pareja que provenía de una unión no heterosexual. En todos los casos, solo uno de los miembros de la pareja procedía de una relación anterior y aportaba hijos a la nueva relación, mientras que el otro miembro no tenía descendencia.

La selección de las personas informantes fue intencional, y los criterios que se tuvieron en cuenta para su inclusión fueron los siguientes: identificarse como no heterosexual, tener hijos y/o hijas procedentes de una relación de pareja previa y, tras la ruptura, haber iniciado una relación con una persona del mismo sexo. Las características sociodemográficas de la muestra se incluyen en la tabla 1 .

El trabajo de campo se desarrolló entre los meses de junio de 2013 y noviembre de 2014. Las familias entrevistadas vivían en ciudades medias de las comunidades autónomas de Andalucía y Extremadura. Para contactar con las personas informantes se siguieron distintas estrategias. En un primer momento, se recurrió a la red de contactos personales de las investigadoras. Para ello, se informó sobre el objetivo del estudio y la posibilidad de participar en el mismo a personas conocidas que respondían al perfil familiar demandado. Así mismo, se envió un correo electrónico a distintas asociaciones y colectivos LGTBI. En ese correo se daba información acerca de la investigación y se solicitaba la colaboración y la participación de los socios y las socias. Por último, las personas informantes fueron proporcionando nuevos contactos, lo que permitió que la muestra se completase mediante una estrategia de «bola de nieve». Este procedimiento posibilitó superar algunos de los inconvenientes que se asocian con poblaciones ocultas, como es el caso de las familias reconstituidas no heteronormativas. Entre estos inconvenientes se encuentran los siguientes: el acceso a la muestra y la participación e implicación en la investigación (Atkinson y Flint, 2001; Bernard, 1995; Heckathorn, 1997).

Como técnica de recogida de datos se empleó la entrevista semiestructurada. Se elaboró un guión en el que aparecían los principales temas a abordar. 
Estos iban de lo más general, principalmente datos de sus biografías personales, a lo más específico. Las entrevistas se realizaron tanto en los domicilios particulares como en espacios públicos. Antes de iniciar la entrevista, se solicitó consentimiento verbal para la grabación y para el uso de la información proporcionada. Se optó por entrevistar de forma conjunta a los dos miembros de la pareja e individualmente cuando uno de los miembros no podía asistir a ella por motivos personales o profesionales. De las entrevistas empleadas en este trabajo, solo una de ellas se realizó de forma individual. Por su parte, las entrevistas conjuntas permitieron superar los posibles conflictos éticos relativos al anonimato y al consentimiento entre las partes de la pareja y, al mismo tiempo, este procedimiento favoreció una mayor riqueza discursiva, tanto en los temas como en los datos proporcionados al crear un espacio para la reflexión conjunta (Bjornholt y Farstad, 2014). Distintos estudios (Taylor y De Vocht, 2011; Torgé, 2013) han puesto de manifiesto las ventajas de las entrevistas conjuntas en investigaciones que versan sobre dinámicas familiares, en concreto las que se refieren al estudio del clima de interacción generado y a los datos que de ahí proceden. No obstante, este tipo de entrevistas también tienen limitaciones que se derivan de las relaciones de poder que prevalecen en algunas parejas, lo que puede provocar que un miembro acapare todo el discurso (Morris, 2001).

Una vez realizadas, las entrevistas fueron transcritas y con posterioridad se procedió al análisis de su contenido. Dicho análisis se realizó desde un enfoque fenomenológico, es decir, se trató de analizar cómo estas familias «se perciben, describen, sienten, juzgan, recuerdan, le dan sentido y hablan sobre sí mismas» (Patton, 2002: 104). Este proceso de análisis se compone de una serie de etapas que tienen por objetivo reducir todo el volumen de datos hasta aproximarse al "fenómeno» de estudio (Finlay, 2014; Sullivan, 2012). Para ello, en un primer momento se identificaron las principales unidades de significado, esto es: «aquellas palabras, frases, comunicación no verbal o paralingüística que expresa un significado único y coherente (independientemente de la pregunta de investigación) y claramente diferenciado de lo que le precede y le sigue» (Hycner, 1985: 282). A continuación, se fueron agrupando las unidades de significado que, en este caso, se trataba de la unidad "Las familias reconstituidas». Y, por encima de esta agrupación, se situó el tema que lleva por nombre «Familias no heteronormativas». Para proceder a esta clasificación de la información, se empleó el programa informático N-Vivo, versión 10, que facilitó la sistematización y la organización de todos los datos procedentes de las entrevistas.

\section{Resultados}

\section{1. "Soy su amiga, no su madre». Percepción del coprogenitor o la coprogenitora en la unidad familiar}

En términos generales, los progenitores y las progenitoras tienden a definir en términos de amistad la relación que las coprogenitoras y los coprogenitores tienen con sus hijos e hijas. Así mismo, los coprogenitores y las coprogenitoras 
también se identifican como amigos de la descendencia de su pareja. Ni unos ni otros se perciben como parientes de ningún tipo o grado. En este sentido, Diego considera que la relación que tiene con el hijo de su pareja, Rodrigo, es de amistad. Él se define como un amigo que le presta apoyo y consejo cuando lo necesita. A través de esa relación de amistad ha sido capaz de ganarse la confianza del hijo de Rodrigo:

Yo soy un amigo. Yo para él era un amigo y sigo siendo un amigo [...] yo siempre he funcionado más como un confidente. De hecho, hay cosas que me las cuenta a mi que no se las cuenta al padre. (Diego, 44 años)

Los progenitores y progenitoras biológicos defienden esa idea de amistad para sus parejas respectivas. De las personas entrevistadas, ninguna mostró interés en que su pareja se identificase o fuese identificada en términos de parentesco. Consideran que la relación de amistad es muy valiosa y que, como señala Diego, permite a los hijos e hijas tener la suficiente seguridad y confianza como para gozar de un vínculo cercano. La ausencia de una estructura jerárquica, propia de las relaciones de parentesco, permite a los hijos e hijas ver, en la pareja de su progenitor o progenitora, a un referente a quien poder acudir para desahogarse, buscar consejo, etc. Para Olga, su compañera, Tamara, se ajusta a ese perfil de amistad que, en ningún momento, roza los lazos de parentesco:

Yo la clasificaría, como te he dicho antes, como una buena amiga. Y eso es lo que yo quiero que sea, su confidente, su amiga, pues que si tiene algún problema pues que ella pueda aconsejarle, que ella pueda ayudarle, que ella pueda..., que si yo no estoy, que sepa que está ella. Poder acudir más a ella. (Olga, 49 años)

Para los coprogenitores y las coprogenitoras la edad de los hijos e hijas de su pareja es un factor que influye en el modo de percibirse y de definirse ante ellos y ellas. Consecuentemente, opinan que el ejercicio de los roles parentales es más difícil cuando se trata de adolescentes o de personas ya adultas que cuando son bebés o criaturas pequeñas. De ahí que la amistad se convierta en la alternativa más factible. Esa idea de amistad repercute en la relación de pareja. A Diego, por ejemplo, le cuesta tener ciertos comportamientos de pareja ante la presencia del hijo de Rodrigo. Cree que si se hubiese criado con ellos desde pequeño estas situaciones no se producirían. Así lo explicaba en la entrevista:

Obviamente, en un principio, para mí era un extraño. Un extraño de dieciséis años, prácticamente un hombre, ya que estaba en casa viviendo contigo o..., y entonces obviamente [...] Pero yo, por ejemplo, ¿no?, a él no le doy besos delante de él, del hijo. Tan solo el treinta y uno de diciembre. Yo, si no, no le doy ningún beso. A lo mejor, viene mi madre y sí le doy un beso delante de ella y no delante de él [...]. Sí, pero es lo que te he dicho. Como no lo he tenido desde chiquitín, no he podido mostrar este tipo de comportamientos normalizados delante de Rodrigo. Que ha sido ya un adulto, prácticamente, 
con el que yo me he encontrado, ni si quiera adolescente, pues a mí me ha dado mucho corte. A él [Rodrigo] no tanto, pero a mí sí. (Diego, 44 años)

La edad también influye en la relación de los hijos e hijas con su coprogenitor o coprogenitora. Es decir, cuando se trata de criaturas pequeñas hay una percepción de estas en términos más próximos al parentesco que a la amistad. Dos de las familias entrevistadas habían iniciado el proceso de reconstitución familiar con hijos e hijas de corta edad. Este el caso de Enrique y Simón que, cuando comenzaron su relación, la hija de Enrique tenía siete años. Ella emplea términos que denotan una relación parental para referirse a Simón y que, en modo alguno, se aproximan al modelo «ideal» de familia heterosexual:

Simón es su madrastra. Le dice su madrastra. «Es mi madrastra» [Entrevistadora: "Y eso, ¿surgió de ella?».] Sí, sí, sí. Vamos a ver, ella dice: «Es mi madrastra». (Enrique, 28 años)

Esta mayor cercanía repercute en una relación más estrecha y cercana entre todos los sujetos de la unidad familiar. En el siguiente verbatim Enrique explica cómo se fue forjando esa relación cercana, de confianza:

Sí que es verdad que nunca nos hemos tapado ni, a lo mejor, a la hora de darnos un beso o cualquier cosa con Simón. Entonces, aparte, yo con mi hija la complicidad, sobre todo eso, la complicidad que tenemos entre los tres. (Enrique, 28 años)

En el momento en que Lorena y Diana iniciaron su relación de pareja, la primera tenía un hijo de tres años. A su vez, la exmujer de Lorena también inició una relación de pareja con otra mujer. En un principio, el niño no aceptaba que Lorena estuviese con Diana. Sin embargo, al poco tiempo comenzó a aceptarla y a llamarla «mamá». Diana habló con él y le dijo que solo tenía una mamá y que, por tanto, a ella debía llamarla por su nombre. Con esto, Diana consiguió marcar los límites acerca de cuál ha de ser la percepción que el hijo de su compañera debía tener sobre ella:

Bueno, de alguna manera, como mami se ha vuelto a casar, pues, se empeñó de que su pareja también era mami del niño. [...]. Y, entonces, yo ahí no estaba de acuerdo. Habían tenido ellas ahí eso y ellas no sé cómo lo llevan pero el hecho es que ella decía que a la otra la llamaba «mami» y no sé qué. Entonces, a mí, en una de las semanas que vino, empezó a llamarme «mamá Diana». Y yo decía: "Yo no soy mamá. Yo soy Diana». Y ya un día me dijo: "pues es que si Josefa [pareja de la exmujer de Lorena] es mi mami, tú eres mi mamá». Claro, razonablemente lógico. Solo que yo le dije: "Josefa no es tu mami ni yo soy tu mamá». Entonces, se enfada con eso, cuando se lo digo. Porque yo lo tengo claro, pero lo tengo claro por él. (Diana, 54 años)

En el contrapunto de esta situación se encuentra el hijo veinteañero de Virginia quien define a Dolores como «la mujer de mi madre». Con esta expresión 
identifica a Dolores a partir del vínculo jurídico que ella tiene con su madre y que, en ningún caso, lo involucra a él como parte de esa definición parental.

\section{2. "Juntos en las misma foto". Relación de las hijas y los hijos con el coprogenitor y la coprogenitora}

En un modelo en el que los coprogenitores y las coprogenitoras se identifican y son identificados como amigos, las funciones que desempeñan son limitadas $y$, bajo ninguna circunstancia, entran dentro de lo que se considera propio de la madre o del padre. En este sentido, la relación y las funciones del coprogenitor o de la coprogenitora van a estar determinadas por factores externos e internos. Entre los factores externos, estarían aquellos que tienen que ver de forma directa con la figura del progenitor o la progenitora. A este respecto, cabe señalar cómo se produjo el divorcio y cuál ha sido la posterior relación entre los progenitores y las progenitoras.

De las cinco familias entrevistadas, solo una de ellas perdió el contacto con uno de los hijos a raíz del divorcio. Se trata del hijo mayor de Rodrigo que decidió dejar de relacionarse con su padre una vez que se divorció e inició su relación de pareja con Diego. Ambos achacan esta circunstancia a la actitud de la exmujer de Rodrigo que, opinan, malmetió en la relación paterno-filial. Sin embargo, este hecho no condicionó la relación del hijo pequeño con su padre:

Yo, en mi caso, estuve varios meses que no podía estar en contacto con mi hijo y, de hecho, con el mayor de ellos no tengo trato, porque la madre lo convenció de tal manera que... Y hubo unos cuantos meses que no pude tener trato ninguno. Directamente, ella fue un poco la que provocó la situación que se estaba creando. (Rodrigo, 52 años)

Cuando existe una buena relación con el excónyuge o la excónyuge se tiende a reforzar el vínculo materno-filial o paterno-filial, y eso repercute en la aceptación del coprogenitor y la coprogenitora. Todas las familias, a excepción de Rodrigo, mantienen una buena relación con su expareja. La hija de Virginia, quien en un primer momento se opuso a la relación que su madre tenía con Dolores, restableció el contacto con su madre cuando vio que estaba ayudando a su padre en un momento crítico. El marido de Virginia estuvo involucrado en un serio problema judicial y ella decidió apoyarlo porque consideraba que era algo que tenía que hacer por su hija y su hijo:

Bueno, pues, a partir de la separación mía, con mi hija fue chungo. De hecho, mi hija ha tenido algunas historias con Dolores. Fatal, fatal, fatal, muy mal. (Entrevistadora: «Y, ¿cómo retomaste la amistad con tu hija?».) A raíz de su padre [...] A él le echaron cárcel. Entró en prisión. Yo iba a prisión porque tenía todos los meses un vis a vis con él, con él y con mis hijos, para apoyarle. La verdad es que, en cierta medida, le daba yo una inyección de moral, porque empezaba yo con las bromas, con las tonterías. Él se ponía a llorar [...] Hombre, yo, la verdad que él lo pasó muy mal. Mis hijos me lo demandaban 
en cierta medida [...] Él, entonces, tuvo que llamar a su hija: «Mira, oye, que me llevan para prisión porque aquí». Ella vino, fuimos en el coche. Y ya a raíz de ahí me llamó, me habló: «Porque mi padre...». (Virginia, 57 años)

Olga también mantiene una buena relación con su exmarido quien está muy presente en la vida familiar. Por su parte, Olga explica que tanto sus hijos como su exmarido aceptan la relación que tiene con Tamara, y esto se constata en que, coincidiendo con la graduación de la hija de Olga, los cinco se hicieron una foto conjunta. Para Olga, esta foto tiene una importancia especial, en la medida en que existe un reconocimiento de lo que representa Tamara en esta nueva etapa de su vida:

Mi hija ha hecho la graduación y mi exmarido ha sido quien nos ha sacado las fotos y, bueno, entre todos, hemos salido en la foto los cinco. Está él, los niños y nosotras dos. También nos ha hecho una foto a los cuatro, de él y yo y los niños, pero, realmente, la que está puesta en el Facebook es la de los cinco, no la de los cuatro. Entiendes, que, dentro de lo que cabe, un respeto de decir pues si es la persona a la que yo quiero, pues que de esa manera, pues que lo sea. Como siempre me ha dicho [su ex marido], vamos. (Olga, 49 años)

Un tercer factor que también involucra al progenitor o a la progenitora y que repercute directamente en la relación con el coprogenitor o la coprogenitora es clarificar su posición ante las hijas y/o los hijos. Cuando el divorcio no ha sido conflictivo, el otro progenitor o progenitora está presente y tiene una implicación fuerte con sus hijos y/o hijas, el coprogenitor o la coprogenitora puede ser visto o vista como una amenaza, como alguien que viene a sustituir a su padre o a su madre. De este modo, los progenitores y las progenitoras que han iniciado una relación de pareja tienden a dejar claro que esta persona no va a sustituir ni a usurpar las funciones de su padre o de su madre. Tras el divorcio, el hijo de Virginia decidió irse a vivir con su madre y Dolores. Tras unos años de convivencia, Virginia y Dolores decidieron casarse. Virginia señalaba que, cuando se lo comentó a su hijo, él mostró cierta disconformidad con la decisión. Ante esta reacción, Virginia le explicó que el matrimonio no iba a cambiar nada, puesto que tanto ella como su padre eran irreemplazables:

Y, entonces, a mi hijo le pregunté: «¿No te alegras?». Se quedó un poco... Dice: «Hombre...». Le digo: «Pero, ¿por qué?, ¿porque es una mujer?». «No, no. Porque te casas.» «Porque me caso, ¿por qué?» «Pues lo mismo me pasaría con mi padre.» Entonces le dije: «Mira, nunca jamás nadie me va a sustituir a mí, ni nadie va a sustituir a tu padre. Son épocas distintas y son personas que tienen una misión y ya está. Y tu padre, supongo yo, que nunca me va a sustituir a mí por nadie. Ni nadie, por muy bueno que sea, va a ser yo». Entonces, bueno, a raíz de ahí parece que cambió un poquito. (Virginia, 57 años)

Cuando Enrique comenzó su relación con Simón también le explicó a su hija que su nueva pareja no iba a reemplazar a su madre. Insistió en que tanto él como su madre eran insustituibles y que, por tanto, no tenía nada que temer. 
Tras superar las dificultades iniciales, Enrique considera que la relación de Simón con su hija se ha ido fortaleciendo:

Y digo: «Tú estás con mamá el tiempo que tengas que estar, y luego estás conmigo. Nosotros siempre vamos a ser tus padres. Que no podemos vivir juntos, que no podemos estar juntos, pero eso no significa que no tal». Y, la verdad, que en ese sentido... Lo que pasa que eso, al principio sí que tenía rechazo de, de que le cogiese la mano, de que diese muestras de cariño con él, pero, claro, porque todos los niños son muy egoístas. Entonces, lo que quieren es su padre y su madre solo para ellos y nadie más. Entonces, bueno, eso fue al principio pero luego ya bien. (Enrique, 28 años)

Junto con estos factores externos al coprogenitor o a la coprogenitora, también se pueden identificar otros de tipo interno que están relacionados con su actitud y con su comportamiento respecto a los hijos y/o las hijas de su pareja. En este sentido, la relación de amistad conlleva no traspasar los límites ni adentrarse en aquellas funciones parentales que no les corresponden. Para Olga, la clave de la convivencia familiar está en que Tamara se mantenga al margen de los problemas que pueda tener con su hija y su hijo. De ahí que Olga se erija como mediadora entre su pareja y sus hijos ante cualquier asunto que pueda ser una fuente de conflictos. Con esta estrategia, Olga trata de limitar la capacidad de mando de Tamara:

Mis hijos son míos. Es que, en realidad..., porque evidentemente mi hija que es adolescente es mucho más complicada. Sí, se lo digo: «Déjamela a mí, déjamela a mí». Primero, porque si yo le digo algo, soy su madre y me lo va a perdonar para los restos de la vida. A ti no. Eso lo primero. Segundo, yo no le he permitido ni el más mínimo comentario. A ti no te lo voy a permitir tampoco. Entonces, no queremos que haya problemas. Tú, mantente al margen. Cosa que no te guste, cosa que veas que es problemática, me las cuentas. Yo la transmito o yo te transmito aquí, o yo hago como intermediaria en esto pasa, esto deja de pasar, o esto no, no debe de pasar. Pero, realmente, la que tengo que educar a mi hija soy yo. [...] Es mi problema y yo tengo que contar con tu ayuda, evidentemente, pero, como se dice, en la trastienda». (Olga, 49 años)

Por su parte, Diego cree que, como coprogenitor, no debe inmiscuirse en la educación del hijo de Rodrigo. Reconoce que él no tiene la autoridad suficiente para educarlo y que, en caso de que lo haga, lo único que puede conseguir es empeorar la situación:

Yo siempre le he dado la responsabilidad de que regañe al hijo, de que hable con el hijo y de que... Yo, cuando he tenido algún problema, le he dicho: «Mira, yo no voy a hablar con él, porque no me ve a mi como una figura paterna». Él va a discutir conmigo, él va a pillar un rebote tremendo conmigo porque no me ve a mí como un padre, ni como ninguna autoridad. Entonces yo siempre decía: «Habla tú con él, porque si yo hablo con él voy a discutir». (Diego, 44 años) 
Los argumentos de Dolores son similares a los de Diego. Ella considera que debe ocupar un «segundo plano» en lo relativo a la relación con el hijo conviviente de Virginia. Desde esa posición, Dolores le comenta a Virginia ciertas cosas que no le agradan de su hijo, pero siempre delegando en la madre para que sea ella quien intervenga y solucione las desavenencias:

La verdad es que yo también me he puesto en un segundo plano con ella y sus hijos porque no quería, realmente, interferir. [...] Y con su hijo fue distinto, porque yo con su hija no vivimos ninguna de las dos, entonces, la relación es distinta. Pero con su hijo hay más convivencia, que le pincho más a su madre porque veo cosas que... Yo no puedo decírselo a su, no debo decírselo a su hijo. O sea, no puedo asumir ningún papel de nada, sino que tiene que ser su madre la que lo asuma. (Dolores, 50 años)

No traspasar los límites de la amistad también supone respetar la relación paterno-filial o materno-filial. En este sentido, para Diana es importante que Lorena y su hijo tengan tiempos y espacios propios, sin su presencia. Esta idea se ve reforzada por el rol materno que Diana identifica con Lorena y del que ella no participa:

Yo lo que pasa es que sí le doy su espacio. O sea, yo entiendo que, y sobre todo a esta edad, él todavía necesita a su mamá. Entonces, pues yo sí que en los ratos que se pone mimoso quiere estar con su mamá, eso está claro. Y yo ese espacio sí que se lo doy. Los veo a los dos ahí acaramelaos... Incluso por las noches yo a lo mejor le cuento un cuento, pero luego ya se queda su mamá, porque ya en ese momento él habla mucho y le cuenta muchas cosas. Y a él le gusta que sea su mamá la que esté ahí con él [...] Aunque yo me lleve bien y eso, su mamá es su mamá. (Diana, 54 años)

Otro factor que repercute en la relación del coprogenitor o la coprogenitora con los hijos y/o las hijas de su pareja está relacionado con lo que, a distintos niveles, esta persona aporta a la unidad familiar. En consecuencia, las progenitoras y los progenitores consideran que sus criaturas valoran que la llegada de la persona coprogenitora es una fuente de ingresos extra y que, ante algún tipo de necesidad económica, puede actuar como un respaldo para el conjunto familiar. En su trabajo, Olga suele tener problemas para cobrar, mientras que Tamara cobra con más puntualidad. Olga comenta que su hija se siente segura con el salario de Tamara, porque saben que ella puede echarles una mano cuando lo necesiten:

Este mes pasado no he cobrado a mi tiempo y mi hija me ha dicho: «Mamá, menos mal que está Tamara con nosotros y ella cobra bien, a su tiempo, y podemos cubrir los gastos del banco». Y yo digo: «Pues sí». Entonces ya empiezan ellos a valorar, aparte del bienestar que a mí me produce, que saben que estoy con una persona nueva, que soy feliz, que se me ve radiante, que se me ve que con ellos también tengo ganas de progresar, de construir. (Olga, 49 años) 
Las madres y los padres señalan que la idea de felicidad y de bienestar que reporta su pareja también es otro de los aspectos valorados por sus hijos e hijas. En la medida en que el coprogenitor y la coprogenitora es una persona importante para su padre o su madre, se sienten satisfechos con su presencia en la vida familiar. De este modo, Virginia explica que su hija le ha comentado que aprecia mucho a Dolores en la medida en que es una persona muy importante para su madre, alguien que le hace sentirse feliz:

Me mandó un whatsapp el día siguiente de casarnos. Íbamos hacia Madrid y me mandó un whatsapp, ¿̇verdad? Que no me había visto en su vida tan feliz, que a Dolores le daba las gracias por lo que había hecho conmigo, que la perdonara. ¡Yo qué sé! Era una cosa... (Virginia, 57 años)

Por último, la existencia de convivencia o no entre el coprogenitor y la coprogenitora y la descendencia de su pareja también determina el tipo de relación. En la muestra, todas las familias tienen hijos y/o hijas convivientes, si bien se puede diferenciar entre aquellas familias que mantienen una convivencia continuada (tres familias) y aquellas otras (dos familias) en las que la convivencia es discontinua, es decir, en función de los regímenes de visita establecidos. En relación con este punto, Dolores reconoce que la convivencia con el hijo de Virginia le obliga a marcar unos límites para evitar conflictos. En cambio, no alude a esa necesidad de que haya límites cuando se refiere a la relación que tiene con la hija no conviviente de su pareja:

Eso es fundamental. Pero no que me respete él a mí, sino yo también a él. Es decir, hay una línea que no podemos pasar ninguno de los dos porque está su madre en el medio. Yo eso lo tengo clarísimo, y siempre se ha evitado pasar esa línea, porque en el momento en que hay una situación en que... Su hijo también es una persona que tiene un carácter fuerte. Intento que, en este sentido, no haya ningún conflicto que esté su madre por el medio y que su madre no tenga que elegir en ningún momento determinado. Además, os lo dije a los dos. Digo: «Que no hay que elegir». O sea, son dos papeles distintos. Ella con su hijo y ella conmigo. Ahora es cierto, yo tengo muchas ganas de que se vaya de casa. (Dolores, 50 años)

\section{Discusión y conclusiones}

El inicio de una relación de pareja con una persona del mismo sexo tras la ruptura de una unión previa (generalmente heterosexual) tiene consecuencias a distintos niveles. Al hilo de los argumentos ofrecidos por las progenitoras y los progenitores, así como por sus parejas respectivas, la opción sexual no parece suscitar mayores conflictos entre las hijas y los hijos que ven como su padre o su madre inicia una relación de pareja con una persona de su mismo sexo. Sin embargo, la idea de conflicto vinculada con la ruptura y con el comienzo de una nueva relación sí está presente en los relatos de las personas informantes. Para prevenir y superar esos posibles conflictos, dichas familias consideran que 
es necesaria una doble negociación: por un lado, la identidad del coprogenitor o la coprogenitora y, por otro, sus funciones en la unidad familiar.

Son varios los factores que interfieren en las negociaciones para identificar quién es y qué debe hacer el coprogenitor y la coprogenitora, entre ellos: la presencia más o menos activa de la madre o del padre no conviviente. En este sentido, María Isabel Jociles y Fernando Villaamil (2008) identifican tres modelos relativos a la posición de la persona coprogenitora en las familias reconstituidas. Estos modelos son el resultado de la combinación del grado de implicación del padre o de la madre no conviviente y del reconocimiento y la asunción de responsabilidades por parte del coprogenitor o de la coprogenitora. El primer modelo es el de «sustitución». En él, los coprogenitores y las coprogenitoras sustituyen a la madre o al padre biológico y los primeros gozan de un amplio reconocimiento por parte de su pareja y de los hijos y/o las hijas de esta. Dicho modelo se produce cuando el padre o la madre no conviviente tiene una escasa o nula implicación en la crianza y en la educación de sus descendientes, y este papel lo acaba asumiendo el coprogenitor o la coprogenitora. En una posición intermedia se encuentra el modelo de "duplicación», en el que tanto el coprogenitor o la coprogenitora como el padre o la madre biológico no conviviente desempeñan las mismas funciones con un grado de implicación similar. Y, por último, Jociles y Villaamil identifican el modelo de «evitación» que destaca porque "el padre y la madre biológicos desempeñan las funciones parentales, y se evita que lo hagan la madrastra y/o el padrastro", así como por una «negación de la posición de "padre o madre" (posición de parentesco) al padrastro o la madrastra» (Jociles y Villaamil, 2008: 217).

Entre las parejas entrevistadas en el presente trabajo, tanto el coprogenitor o la coprogenitora como su pareja y sus hijas y/o hijos identifican a dicha persona como un amigo o una amiga. Siguiendo la propuesta de Jociles y Villaamil, estas familias se ubican en un modelo de evitación en el cual las madres y los padres son quienes asumen todo el peso de la maternidad y de la paternidad, mientras que el coprogenitor o la coprogenitora se sitúa en una posición secundaria, con una identidad y unos roles más cercanos a la amistad que al parentesco. Es lo que Beatriz Moncó y Ana María Rivas denominan como una sensación de "lejanía próxima», es decir, a pesar de ser identificadas como personas muy importantes y significativas en la vida de los miembros de la unidad familiar, no son capaces de superar «ese agujero negro que supone el término de amistad» (Moncó y Rivas, 2007: 7). Esa idea de amistad es la que subyace en la mayor parte de las familias reconstituidas no heteronormativas, tanto en el caso español (Rivas, 2008) como en el estadounidense (Hequembourg, 2004; Lynch, 2005; Wright, 2011) o el británico (Tasker, 2013).

La definición del coprogenitor o de la coprogenitora en términos de amistad también está condicionada por la edad de las hijas y/o de los hijos de la pareja. En este sentido, cuanto más jóvenes son los descendientes, mayor es el grado de implicación del coprogenitor o de la coprogenitora en su vida cotidiana y, en consecuencia, mayor es la probabilidad de que su relación se identifique como más próxima al parentesco. En cambio, cuando las hijas y/o los hijos son ado- 
lescentes o adultos resulta más costoso aceptar la nueva situación y, por tanto, crear un vínculo familiar fuerte. Estos resultados coinciden con los hallados por Janet Wright (2011) en su investigación desarrollada en Estados Unidos con familias reconstituidas no heteronormativas. No obstante, bajo la etiqueta de la amistad, el coprogenitor o la coprogenitora se convierte en un referente a quien poder acudir en busca de ayuda o de asesoramiento, en particular cuando se trata de aquellos temas que les resultan más difíciles de abordar con sus madres o sus padres biológicos. Esta percepción que convierte a la persona coprogenitora en una consejera es congruente con los resultados obtenidos en otros estudios procedentes del ámbito estadounidense (Current-Juretschko y Bigner, 2005; Wright, 2011). Como subrayan las británicas Fiona Tasker y Susan Golombok:

Diferentes investigaciones han subrayado la importancia de la comadre o progenitora no gestante en las familias encabezadas por lesbianas. Los hijos adolescentes de las madres lesbianas divorciadas se describen como más abiertos comunicativamente con sus madres y con las actuales parejas de sus madres de lo que se muestran los hijos adolescentes de las parejas heterosexuales divorciadas. (Tasker y Golombok en Tasker y Patterson, 2007: 15)

Así mismo, entre las personas informantes se ha constatado que, cuando en la unidad familiar hay niños y/o niñas, las relaciones adquieren un tono «cuasiparental» (Moncó y Rivas, 2007) que no se produce cuando se trata de adolescentes o de personas adultas. Aún siendo definidos como amigos o amigas, los coprogenitores y las coprogenitoras son identificados como personas significativas en sus vidas.

Por otra parte, la ausencia de un «estatus» propio (Current-Juretschko y Bigner, 2005: 3) o de un "guión" en el que se identifiquen y se definan cuáles deben ser sus funciones (Hequembourg, 2004: 747) coloca a los coprogenitores y a las coprogenitoras en una posición vulnerable. Esta vulnerabilidad obliga a una negociación continua de sus roles, con el fin de adaptarse a unas dinámicas familiares preestablecidas (Brown y Perlesz, 2008; Hequembourg y Farrell, 1999).

De este modo, la negociación de los roles implica tener en cuenta qué hacer y qué no con los hijos y/o con las hijas de su pareja partiendo de cuál es el grado de implicación del progenitor o la progenitora no conviviente (Hequembourg, 2004). En consecuencia, cuando el coprogenitor y la coprogenitora ha sido definido en términos de amistad, es preciso que no traspase los límites de lo que socialmente se espera y se desea de un amigo o de una amiga. Ejercer ciertas funciones o asumir determinadas responsabilidades puede provocar malestar entre el padre o la madre, al considerar que su pareja se está inmiscuyendo más de la cuenta en asuntos que no son de su competencia (CrosbieBurnett y Hehlmbrecht, 1993; Rivas, 2008; Wright, 2011). Por tanto, dentro de este modelo de evitación, la progenitora o el progenitor prefiere el consejo de su pareja antes que su participación e implicación activa en aquellas cuestiones que afectan de modo directo a su relación materno-filial o paterno-filial 
(Wright, 2011). El coprogenitor y la coprogenitora es la persona a quien se puede acudir en busca de consejo o ayuda, pero no quien imponga y haga cumplir las normas.

Además, traspasar estos límites no solo es definido como una intromisión en asuntos a los que deberían permanecer ajenos, sino también como un intento de sustituir al padre o a la madre no conviviente. Como han señalado las autoras estadounidenses Pauline Erera y Karen Fredriksen (2001), una de las posibles fuentes de conflicto familiar por parte de los hijos y/o de las hijas tiene que ver con la percepción del coprogenitor o de la coprogenitora como una persona sustituta de su padre o de su madre, según los casos. Dentro de un entramado de relaciones familiares en el que la figura de este otro progenitor o progenitora está muy presente, dichas cuestiones se convierten en asuntos de primer orden que son mirados muy de cerca por todos los miembros de la unidad familiar. En este sentido, todas las parejas entrevistadas destacan que es necesario que los coprogenitores y las coprogenitoras se mantengan en la posición negociada para, de este modo, garantizar la convivencia.

Ante los inconvenientes y los problemas que surgen a raíz de la convivencia, las familias reconstituidas no heteronormativas tienden a buscar un equilibrio entre la relación con la pareja y con las hijas y/o los hijos en un contexto en el que es posible que "cada uno quiere ser el primer "otro" para el progenitor biológico" (Lynch, 2005: 53) y en donde dichas relaciones se perciben como «opuestas, excluyentes y rivales» (Rivas, 2008: 194). Ese equilibrio pasa, pues, por dar la respuesta correcta que satisfaga las necesidades de su pareja y la de sus descendientes, teniendo en cuenta las circunstancias específicas y los límites de uno y otros (Current-Jaretschko y Bigner, 2005; Wright, 2011).

La ruptura con la heteronormatividad social en las relaciones familiares implica deconstruir roles, estereotipos y funciones sociales que hacen tambalear las concepciones de "la familia», así como la normatividad de género. De este modo, las familias reconstituidas no heteronormativas tienen que buscar fórmulas que les permitan legitimar sus relaciones y dotarlas de significado. El recurso a la amistad, y las funciones que esta institución lleva asociadas, se convierte en una opción que les permite solventar dichas vicisitudes.

\section{Referencias bibliográfícas}

AtKInson, Rowland y Flint, John (2001). «Accessing hidden and hard to reach populations: Snowball research strategies». Social Research Update, 33. <http://sru.soc.surrey.ac.uk/SRU33.pdf>.

BECK-GERNSHEIM, Elisabeth (2002). Reinventing the family: In search of new lifestyles. Cambridge: Polity Press.

BERGER, Roni (2000). «Gay stepfamilies: a triple-stigmatized group». Family in Society, 81 (5), 504-516. $<$ https://doi.org/10.1606/1044-3894.1052>

BERKOWITZ, Dana y MARSIGLIO, William (2007). «Gay men: Negotiating procreative, father, and family identities». Journal of Marriage and Family, 69 (2), 366-381. $<$ https://doi.org/10.1111/j.1741-3737.2007.00371.x> 
BERnARD, Russell (1995). Research methods in anthropology. California: AltaMira Press. BJORNHOLT, Margunn y FARSTAD, Gunhild (2014). «Am I rambling?: On the advantages of interviewing couples together». Qualitative Research, 14 (3), 3-19. <https://doi.org/10.1177/1468794112459671>

BlaCKWOOD, Evelyn y WiERINGA, Saskia (2003). «Sombras sáficas: Desafiando el silencio sobre el estudio de la sexualidad». En: NieTo, José Antonio (ed.). Antropología de la sexualidad y diversidad cultural. Madrid: Agora.

BROWN, Rhonda y PERLESZ, Amaryll (2008). "In search of a name for lesbians who mother their non-biological children». Journal of GLBT Family Studies, 4 (4), 453-467. <https://doi.org/10.1080/15504280802191574>

CAdoret, Anne (2013). Padres como los demás: Parejas gays y lesbianas con hijos. Barcelona: Gedisa.

Crosbie-Burnett, Margaret y HeHlmbrecht, Lawrence (1993). «A descriptive empirical study of gay male stepfamilies». Family Relations, 42 (3), 252-262. <https://doi.org/10.2307/585554>

CUCCHIARI, Salvatore (2000). «La revolución de género y la transición de la horda bisexual a la banda patrilocal: Los orígenes de la jerarquía de género». En: LAMAS, M. (ed.). El género: La construcción cultural de la diferencia sexual. México: Grupo Editorial Miguel Ángel Porrúa.

Current-JuretSCHKO, Lisa y BigneR, Jerry (2005). «An Exploratory Investigation of Gay Stepfathers' Perceptions of Their Role». Journal of GLBT Family Studies, 1 (4), 1-20. <https://doi.org/10.1300/j461v01n04_01>

DONOVAN, Catherine (2000). "Who needs a father?: Negotiating biological fatherhood in British lesbian families using self-insemination». Sexualities, 3 (2), 149-164. <https://doi.org/10.1177/136346000003002003>

ERERA, Pauline y FredrIKSEN, Karen (2001). «Lesbian stepfamilies: A unique family structure». En: LEHMANN, Jennifer (ed.). The gay \& lesbian marriage \& family reader. Nebraska: Gordian Knot Books.

FINLAY, Linda (2014). «Engaging phenomenological analysis». Qualitative Research in Psychology, 11 (2), 121-141. <https://doi.org/10.1080/14780887.2013.807899>

HeckATHorn, Douglas (1997). «Respondent -driven sampling: A new approach to the study of hidden populations». Social Problems, 44 (2), 174-199. <https://doi.org/10.2307/3096941>

Hequembourg, Amy (2004). "Unscripted motherhood: Lesbian mothers negotiating incompletely institutionalized family relationships». Journal of Social and Personal Relationships, 21, 739-762. <https://doi.org/10.1177/0265407504047834>

Hequembourg, Amy y Farrell, Michael (1999). «Negotiating marginal-mainstream identities». Gender and Society, 13 (4), 540-557. <https://doi.org/10.1177/089124399013004007>

HERrERA, Florencia (2009). «Tradition and transgression: Lesbian motherhood in Chile». Sexuality Research and Social Policy, 6 (2), 35-51. <https://doi.org/10.1525/srsp.2009.6.2.35>

HYCNER, Richard (1985). «Some guidelines for the phenomenological analysis of the interview data». Human Studies, 8, 279-303. <https://doi.org/10.1007/bf00142995> 
ImAZ, Elixabete (2006). «La maternidad en el seno de las parejas lesbianas: Cambios, continuidades y rupturas respecto a los modelos familiares y maternales». Arxius de Ciències Socials, 15, 89-100.

InGRAHAM, Chrys (1994). "The heterosexual imaginary: Feminist sociology and theories of gender». Sociological Theory, 12 (2), 203-219. <https://doi.org/10.2307/201865>

Instituto NaCiOnal de Estadística (2011). Censo de población y viviendas 2011. <http://www.ine.es/censos2011_datos/cen11_datos_inicio.htm>.

- (2011). Encuesta continua de hogares. <http://www.ine.es/prensa/np965.pdf>.

JOCILES, María Isabel y VILAAMIL, Fernando (2008). «Estrategias de sustitución en la construcción de la paternidad y la maternidad dentro de las familias reconstituidas». Papers: Revista de Sociología, 90, 213-240. $<$ https://doi.org/10.5565/rev/papers/v90n0.743>

LEHR, Valerie (1999). Queer family values: Debunking the myth of the nuclear family. Filadelfia: Temple University Press.

LÓPEZ, Francisca (2014). Experiencia vital, perfil psicológico y orientación sexual de jóvenes adultos con madres lesbianas o padres gays. Sevillla: Universidad de Sevilla. Tesis doctoral.

LYNCH, Jean (2005). «Becoming a stepparent in gay/lesbian stepfamilies». Journal of Homosexuality, 48 (2), 45-60. <https://doi.org/10.1300/j082v48n02_03>

LYNCH, Jean y MURRAY, Kim (2000). «For the love of the children: The coming out process for lesbian and gay parents and stepparents». Journal of Homosexuality, 39 (1), 1-24. <https://doi.org/10.1300/j082v39n01_01>

Mallon, Gerald (2004). Gay men choosing parenthood. Nueva York: Columbia University Press.

Mezey, Nancy (2008). New choices. New families: How lesbians decide about motherhood. Baltimore: John Hopkins University Press.

Moncó, Beatriz y RIVAS, Ana María (2007). «La importancia de "nombrar”: El uso de la terminología de parentesco en las familias reconstituidas». Gazeta de Antropología, 23.

MORRIS, Sara (2001). "Joint and individual interviewing in the context of cancer». Qualitative Health Research, 11, 553-567.

PATTERSON, Charlotte (2000). "Family relationships of lesbian and gay men». Journal of Marriage and the Family, 62 (4), 1052-1069. <https://doi.org/10.1111/j.1741-3737.2000.01052.x>

PATTON, Michael (2002). Qualitative research \& evaluation methods. California: SAGE.

PICHARDO, José Ignacio (2008). Opciones sexuales y nuevos modelos familiares. Madrid: Universidad Autónoma de Madrid. Tesis doctoral.

- (2009). «(Homo)sexualidad y familia: Cambios y continuidades al inicio del tercer milenio». Politica y Sociedad, 46 (1-2), 143-160.

- (2011). «We are family (or not): Social and legal recognition of same-sex relationships and lesbian and gay families in Spain». Sexualities, 14 (5), 544-561. <https://doi.org/10.1177/1363460711415217>

Real Academia Española (2001). Diccionario de la lengua española. 22. a ed. $<$ http://www.rae.es/rae.html>.

RivaS, Ana María (2008). «Las nuevas formas de vivir en familia: El caso de las familias reconstituidas». Cuadernos de Relaciones Laborales, 26 (1), 179-202. 
Rivas, Ana María (2012). «El ejercicio de la parentalidad en las familias reconstituidas». Portularia, 12 (2), 29-41. <https://doi.org/10.5218/prts.2012.0042>

Sullivan, Paul (2012). Qualitative data analysis: Using a dialogical approach. Londres: SAGE.

TASKER, Fiona (2013). «Lesbian and gay parenting post-heterosexual divorce and separation». En: Goldberg, Abbie E. y Allen, Katherine R. (eds.). LGBT-Parent families: Innovations in research and implications for practice. Nueva York: Springer.

TASKER, Fiona y PATTERSON, Charlotte (2007). «Research on gay and lesbian parenting». Journal of GLBT Family Studies, 3 (2-3), 9-34.

TAYLOR, Bridget y DE VOCHT, Hilde (2011). «Interviewing separately or as couples?: Considerations of authenticity of method». Qualitative Health Research, 21, 1576-1587.

TorGÉ, Cristina Joy (2013). «Using conjoint interviews with couples that have been living with disabilities and illnesses for a long time: Implications and insights». Qualitative Studies, 4 (2), 100-113.

WARNER, Michael (2001). Fear of a queer planet: Queerpolitics and social theory. Minneapolis: University of Minnesota Press.

WeSTON, Kath (2003). Las familias que elegimos: Lesbianas, gays y parentesco. Barcelona: Edicions Bellaterra.

WOLKOMIR, Michelle (2009). «Making heteronormativity reconciliations: The story of romantic love, sexuality, and gender in mixed-orientation marriages». Gender and Society, 23, 494-519.

<https://doi.org/10.1177/0891243209340033>

Wright, Janet (2011). Lesbian step families: An ethnography of love. Nueva York: Routledge. 